\title{
Measurement of Gamma-Ray from Inelastic Neutron Scattering on ${ }^{56} \mathrm{Fe}$
}

WANG Zhaohui ${ }^{1, *}, R E N \mathrm{Jie}^{1}$, WU Hongyi ${ }^{2}$, QIAN Jing ${ }^{1}$, HUANG Hanxiong ${ }^{1}$, WANG Wenming ${ }^{1}$, JIANG Wei $^{3,4}$, WU Xiaoguang ${ }^{1}$, ZHANG Qiwei ${ }^{1}$, YI $\mathrm{Han}^{3,4}$, WANG $\mathrm{Qi}^{1}$, LI Xia ${ }^{1}$, BAO Jie ${ }^{1}$, RUAN Xichao ${ }^{1}, J_{N} G \mathrm{Hantao}^{3,4}$, TANG Jingyu $^{3,4}$,

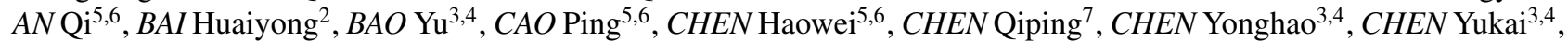
CHEN Zhen ${ }^{5,6}$, CUI Zengqi ${ }^{2}$, FAN Ruirui ${ }^{3,4,5}$, FENG Changqing ${ }^{5,6}$, GAO Keqing ${ }^{3,4}, G U$ Minhao $^{3,5}$, HAN Changcai ${ }^{8}$, HAN Zijie ${ }^{7}, H E$ Guozhu $^{1}, H E$ Yongcheng $^{3,4}, H O N G$ Yang $^{3,4,9}, H U A N G$ Weiling $^{3,4}, H U A N G \mathrm{Xiru}^{5,6}, J I \mathrm{Xiaolu}^{3,5}, J I$ Xuyang ${ }^{5,10}$, JIANG Haoyu ${ }^{2}$, JIANG Zhijie ${ }^{5,6}, K A N G$ Ling $^{3,4}, K A N G$ Mingtao $^{3,4}, L I \mathrm{Bo}^{3,4}, L I \mathrm{Chao}^{5,6}, L I \mathrm{Jiawen}^{5,10}, L I$

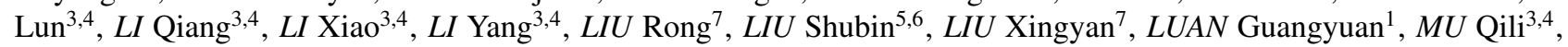
NING Changjun ${ }^{3,4}$, QI Binbin ${ }^{5,6}$, REN Zhizhou ${ }^{7}$, SONG Zhaohui ${ }^{8}$, SONG Yingpeng ${ }^{3,4}$, SUN Hong ${ }^{3,4}$, SUN Kang ${ }^{3,4,9}$, SUN Xiaoyang ${ }^{3,4,9}$, SUN Zhijia ${ }^{3,4,5}$, TAN Zhixin ${ }^{3,4}$, TANG Hongqing ${ }^{1}$, TANG Xinyi ${ }^{5,6}$, TIAN Binbin ${ }^{3,4}$, WANG Lijiao ${ }^{3,4,9}$, WANG Pengcheng ${ }^{3,4}$, WANG Taofeng ${ }^{11}, W E N$ Jie $^{7}, W E N Z_{\text {Zhongwei }}{ }^{7}, W U$ Qingbiao $^{3,4}, W U \mathrm{Xuan}^{3,4}$, XIE Likun ${ }^{5,10}, Y A N G$

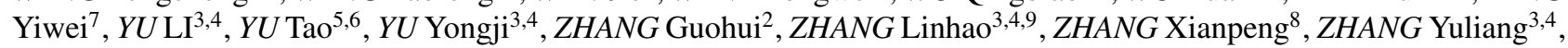
ZHANG Zhiyong ${ }^{5,6}$, ZHAO Yubin ${ }^{3,4}$, ZHOU Luping ${ }^{3,4,9}$, ZHOU Zuying ${ }^{1}$, ZHU Danyang ${ }^{5,6}$, ZHU Kejun ${ }^{3,5}$, and ZHU Peng $^{3,4}$

${ }^{1}$ Key Laboratory of Nuclear Data, China Institute of Atomic Energy, Beijing 102413, China

${ }^{2}$ State Key Laboratory of Nuclear Physics and Technology, School of Physics,Peking University, Beijing 100871, China

${ }^{3}$ Institute of High Energy Physics, Chinese Academy of Sciences (CAS), Beijing 100049, China

${ }^{4}$ Spallation Neutron Source Science Center, Dongguan 523803, China

${ }^{5}$ State Key Laboratory of Particle Detection and Electronics

${ }^{6}$ Department of Modern Physics, University of Science and Technology of China, Hefei 230026, China

${ }^{7}$ Institute of Nuclear Physics and Chemistry, China Academy of Engineering Physics, Mianyang 621900, China

${ }^{8}$ Northwest Institute of Nuclear Technology, Xi' an 710024, China

${ }^{9}$ University of Chinese Academy of Sciences, Beijing 100049, China

${ }^{10}$ Department of Engineering and Applied Physics, University of Science and Technology of China, Hefei 230026, China

${ }^{11}$ Beihang University, Beijing 100083, China

\begin{abstract}
In nuclear reactors, inelastic neutron scattering is a significant energy-loss mechanism which has deep impacts on designments of nuclear reactor and radiation shielding. Iron is an important material in reactor. However, for the existing nuclear data for iron, there exists an obvious divergence for the inelastic scattering cross sections and the related gamma production sections. Therefore the precise measurements are urgently needed for satisfying the demanding to design new nuclear reactors (fast reactors), Accelerator Driven Subcritical System (ADS), and other nuclear apparatus. In this paper, we report a new system with an array of HPGe detectors, electronics and acquisition system. Experiments had been carried out on three neutron facilities.
\end{abstract}

\section{Introduction}

The cross sections of inelastic neutron scattering on ${ }^{56} \mathrm{Fe}$ are important nuclear data for designments of nuclear reactors, Accelerator Driven Sub-critical System (ADS), and other nuclear apparatus. Traditionally, in many experimental measurements, many gamma production cross sections are normalized according to the $847 \mathrm{keV} \gamma$ ray from ${ }^{56} \mathrm{Fe}\left(2^{+}->0^{+}\right)$transition, which makes the precision of the cross sections very critical.[1]

We built experimental setup on three neutron facilities, the CIAE Cockcraft-Walton Accelerator, CIAE $2 \times 1.7 \mathrm{MV}$ accelerator, and Back-n white neutron source at CSNS, and then carried out experiment measurements. The shielding and collimator had been established. Four energies, which

*e-mail: ngamma@163.com are $2.96 \mathrm{MeV}$ and $14.80 \mathrm{MeV}$ at Cockcraft-Walton Accelerator, and $1.2 \mathrm{MeV}$ and $2.5 \mathrm{MeV}$ at $2 \times 1.7 \mathrm{MV}$ accelerator have been measured. And we will continue the studies on CSNS for continuous energy range in near future. We studied the prompt gamma-ray method and obtained the gamma-ray production cross-sections from inelastic neutron scattering on ${ }^{56} \mathrm{Fe}$. The measurement uncertainty is less than $10 \%$.

\section{Experiment on Cockcraft-Walton Accelerator}

Pulsed and continuous deuterium beam experiments have been carried out on Cockcraft-Walton Accelerator, at China Institute of Atomic Energy (CIAE). By using $\mathrm{D}(\mathrm{d}, \mathrm{n}){ }^{3} \mathrm{He}$ and $\mathrm{T}(\mathrm{d}, \mathrm{n}){ }^{4} \mathrm{He}$ reactions, $2.96 \pm 0.26 \mathrm{MeV}$ and 
$14.80 \pm 0.44 \mathrm{MeV}$ neutrons are produced. Figure 1 shows the experimental scheme. An array of High-purity Germanium (HPGe) detectors, as well as the following electronics and acquisition system, are used to measure the prompt $\gamma$ rays from $(\mathrm{n}, \mathrm{xn})$ reaction. The detection system consists of four CLOVER HPGe detectors and four planar HPGe detectors which are located at $110^{\circ}, 150^{\circ}$ and $125^{\circ}$ to the neutron beam direction. Figure 2 shows the layout of the detectors, which is visualized by a Monte Carlo code Geant4.

The old experimental hall of CIAE Cockcraft-Walton

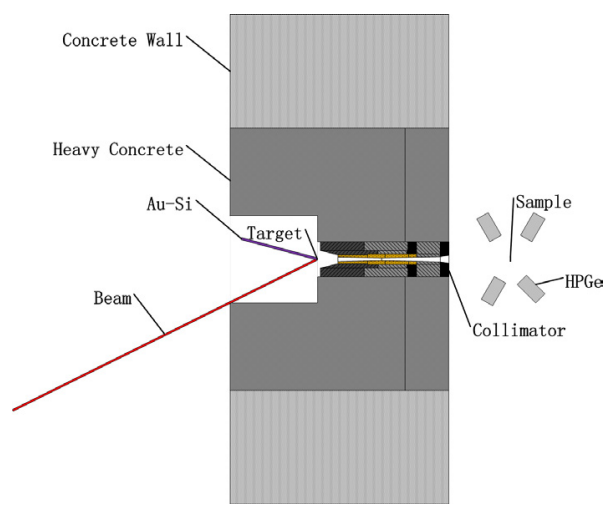

Figure 1. Experimental setup diagram on Cockcraft-Walton Accelerator

Accelerator was modified to carry out the current experiment. The concert wall was increased from $1 \mathrm{~m}$ to $1.5 \mathrm{~m}$; an extra $2 \mathrm{~cm}$ lead shielding was added. Collimators which were made from copper, polyethylene (PE), and lead, are used in this experiment. Two natural iron samples, with size of $\Phi 40 \mathrm{~mm} \times 3 \mathrm{~mm}$ (for $2.96 \mathrm{MeV}$ neutron beams) and $33 \mathrm{~mm} \times 33 \mathrm{~mm} \times 1 \mathrm{~mm}$ (for $14.80 \mathrm{MeV}$ neutron beams), are used in our measurement. They are mounted on an aluminum target holder. At sample position, the neutron beam intensities are $\sim 7000 \mathrm{n} / \mathrm{s} / \mathrm{cm}^{2}(14.80 \mathrm{MeV}$, pulsed), and $\sim 900 \mathrm{n} / \mathrm{s} / \mathrm{cm}^{2}$ (2.96 MeV, continuous) respectively.

The experimental results show that several characteris-

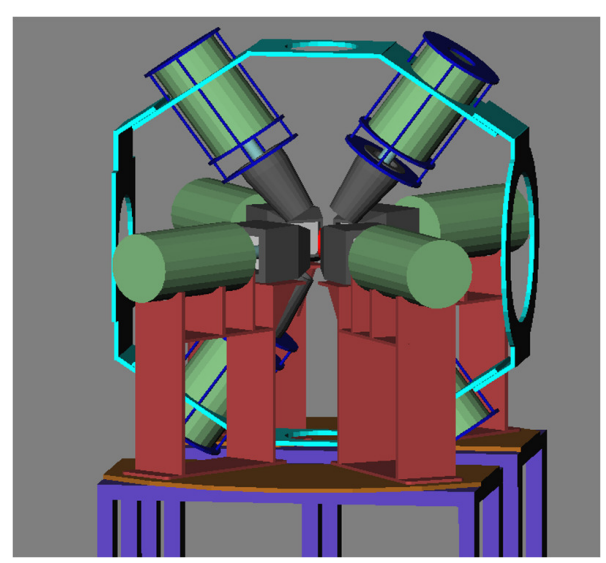

Figure 2. Arrangement of four CLOVER and four planar HPGe detectors

tic $\gamma$-ray peaks of $(n, x n)$ reaction are observed. The ab- solute neutron flux value can be monitored by associated particle measurement, and then given by associated particle method, Therefore the cross sections given here are not obtained relatively. The results will be shown in Sec.4 together with CIAE $2 \times 1.7 \mathrm{MV}$ accelerator data.

\section{Experiment on $2 \times 1.7$ MV Tandem Accelerator}

At CIAE $2 \times 1.7 \mathrm{MV}$ accelerator, neutron beams with energies of $1.20 \pm 0.07 \mathrm{MeV}$ and $2.45 \pm 0.26 \mathrm{MeV}$ can be produced by $\mathrm{T}(\mathrm{p}, \mathrm{n})^{3} \mathrm{He}$ reactions. The neutron shielding is composed of $\mathrm{PE}$ with $20 \% \mathrm{BC}_{4}$, and the neutron collimator is made of copper and lead. The target is also natural iron with size of $\Phi 30 \mathrm{~mm} \times 3 \mathrm{~mm}$. Another target is natural chromium with the same size. At sample position, the neutron beam intensities are $\sim 700 \mathrm{n} / \mathrm{s} / \mathrm{cm}^{2}$. The prompt gamma-ray was measured by four CLOVER detectors placed at $110^{\circ}$ and $150^{\circ}$.

We analyzed the prompt gamma-ray and obtained the gamma-ray production cross sections of ${ }^{56} \mathrm{Fe}\left(\mathrm{n}, \mathrm{n}^{\prime} \gamma\right)$ reaction. The cross sections are obtained by using two methods, one is relative method, i.e. comparing with ${ }^{52} \mathrm{Cr}$ cross section, and another one is the absolute method, i.e. by using absolute neutron flux value.

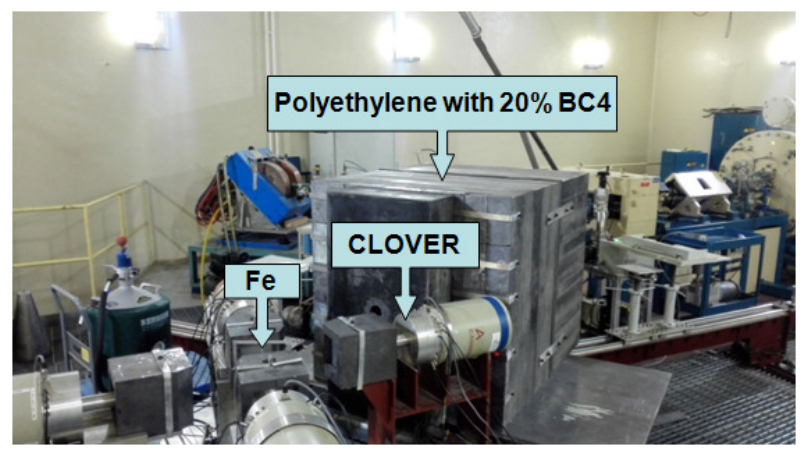

Figure 3. Experimental deployment on $2 \times 1.7$ MV Tandem Accelerator

\section{Data analysis and Results}

The data sets, the one from Cockcraft-Walton Accelerator and one from $2 \times 1.7 \mathrm{MV}$ Tandem Accelerator, are analyzed. The procedure is following: gamma spectrum analysis, incident neutron energy valuation, detection efficiency calibration and so on. The major correction factor include: background influence, neutron flux attenuation and multi-scattering in sample, gamma self-absorption and dead time. The ${ }^{56} \mathrm{Fe}\left(\mathrm{n}, \mathrm{n}^{\prime} \gamma\right)$ cross sections results for neutron incident energies of 1.25, 2.45, 2.96 and $14.80 \mathrm{MeV}$ are shown in Fig.4. We compare the cross sections results with these from Nelson group and Negret group. The cross sections are consistent with their results.[2][3]

By these experiments, we studied the prompt gamma-ray method and give out the gamma-ray production cross sections of ${ }^{56} \mathrm{Fe}$ inelastic neutron scattering. The measure- 
ment uncertainty is less than $10 \%$. The measuring method was verified to be feasible by iron target experiment.

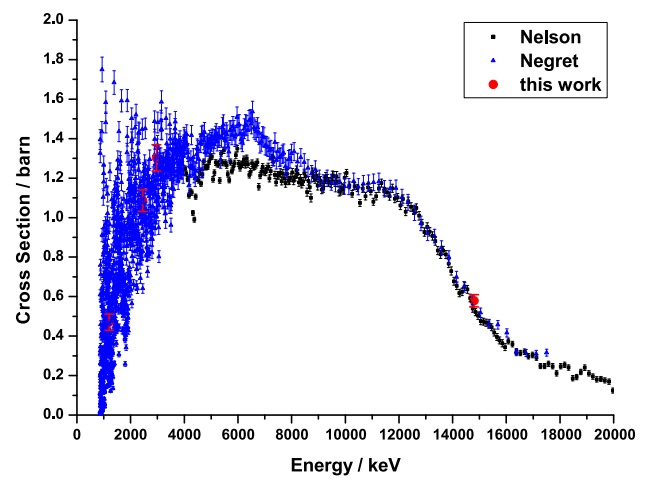

Figure 4. Cross sections comparision with Nelson and Negret

\section{Experiment on China Spallation Neutron Source}

The China Spallation Neutron Source (CSNS) is the first pulsed spallation neutron source in China. It was completed in 2018 and has been running since then. Now, the facility is operating stably at a repetition rate of $25 \mathrm{~Hz}$ at beam power of $100 \mathrm{~kW}$. The Back-n white neutron source is a back-streaming neutrons beamline from the spallation target at CSNS, which can provide neutron beam energies range from $\mathrm{eV}$ to hundreds of $\mathrm{MeV}$.[4] [5]

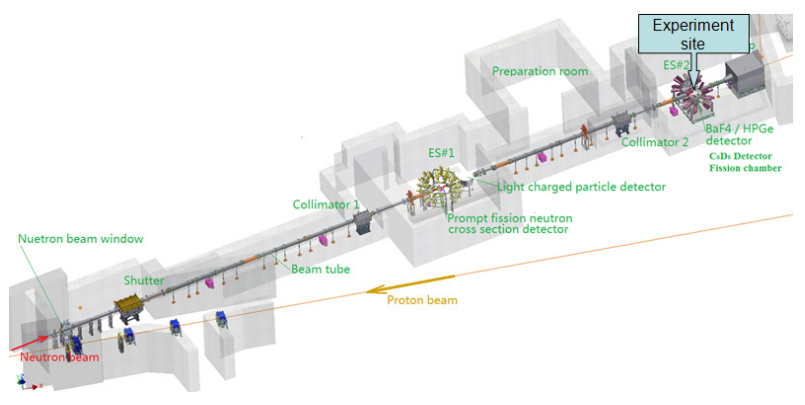

Figure 5. Back-n white neutron beamline and experiment site at CSNS

Our experiment, as the first neutron experiment using HPGe detectors on CSNS, was carried out at CSNS in February, 2019. The experimental location is at the End Station 2 (ES \#2) of the Back-n white neutron source, about $77 \mathrm{~m}$ from the spallation neutron target. The TOF (Time-of-Flight) method was used to determine the neutron energy. However, because the CSNS was working with the double-bunch mode, the TOF spectra are blurred. The double-bunch mode is that during it running there are two proton bunches with time interval of $410 \mathrm{~ns}$ in each pulse. To extract the event distribution corresponding to a single proton bunch, we developed the unfolding methods to obtain higher time resolution and then neutron energy resolution spectra.[6]

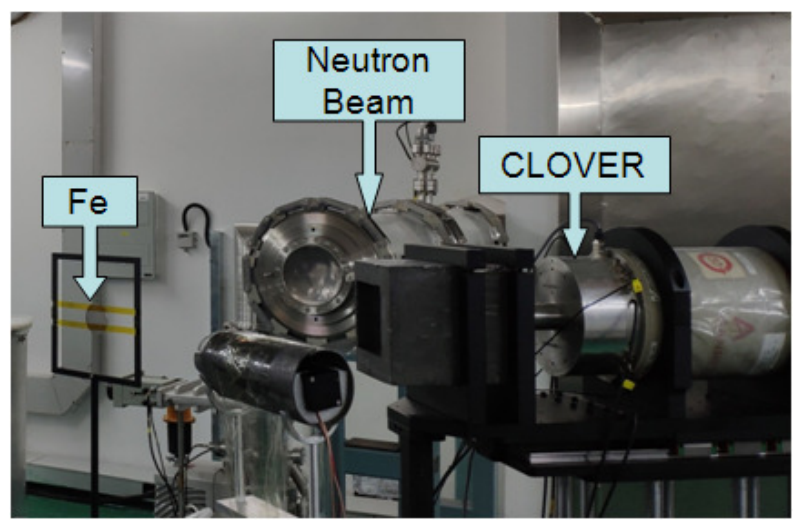

Figure 6. Experimental deployment on Back-n white neutron beamline

The experimental setup is shown in Fig.5. A CLOVER detector was placed at $125^{\circ}$ with respect to the incoming neutron beam to measure prompt gamma-ray. We also employed $\mathrm{LaBr}_{3}$ detector to survey the feasibility of experimental condition. The natural iron sample is a disk with a diameter of $50 \mathrm{~mm}$ and a thickness of $1 \mathrm{~mm}$. The cross section can be deduced by relatively comparing with measurement effect for ${ }^{52} \mathrm{Cr}$ sample.

Figure 7 shows the TOF spectra of the CLOVER detector with the CSNS working on the double-bunch mode. There are two distinct $\gamma$ flash peaks on the TOF spectrum, which means the influence of double-bunch mode is significant for inelastic neutron scattering experiment. Figure 8 shows the gamma spectra for TOF between $2027 \mathrm{~ns}$ and $2068 \mathrm{~ns}$. The $847 \mathrm{keV} \gamma$ ray is obviously on the spectra. It comes from inelastic neutron scattering on ${ }^{56} \mathrm{Fe}$. For double-bunch mode, the unfolding methods will be applied to extract the event distribution corresponding to a single proton bunch. The related data analysis is ongoing.

Figure 9 shows our preliminary result for CSNS Back$\mathrm{n}$ experiment. We compare the $\gamma$ emissivity ratio of $\mathrm{Fe}-$ $847 \mathrm{keV}$ and $\mathrm{Cr}-1434 \mathrm{keV}$ with the corresponding data of GELINA. The normalized emissivity ratio is equal to cross section ratio. So we overlay the cross section of GELINA according to the double-bunch condition of CSNS. The $\mathrm{X}$ axis is TOF on the bottom as well as double-bunch neutron energy on the top. The $\mathrm{Y}$ axis is the $\gamma$ emissivity ratio. From this figure, we can find out that the emissivity ratio is consistent on the whole TOF time range and the data curve shape is similar. Through the data comparison, we can infer that our system is reliable. More accurate analysis will be carried out. 


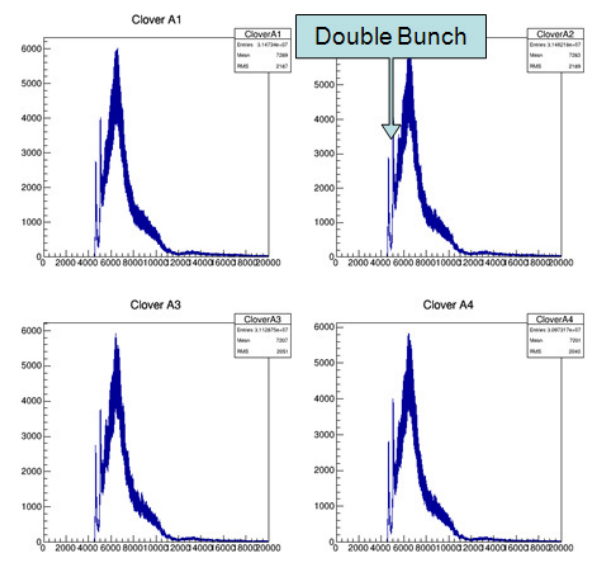

Figure 7. Time-of-Flight spectra of the CLOVER detector

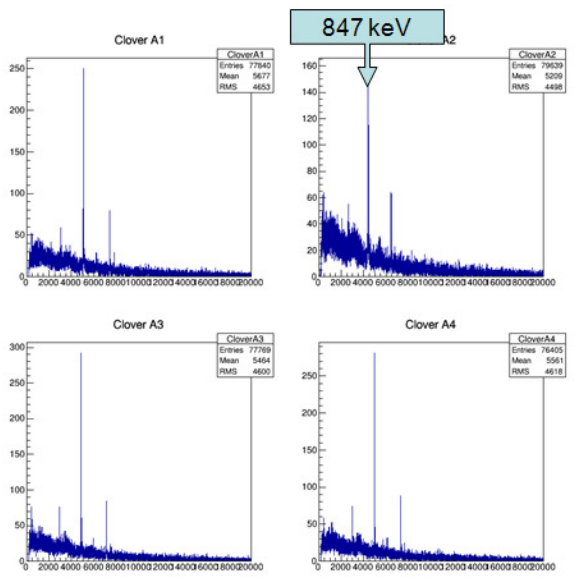

Figure 8. Gamma spectra for TOF between 2027 ns and 2068 ns

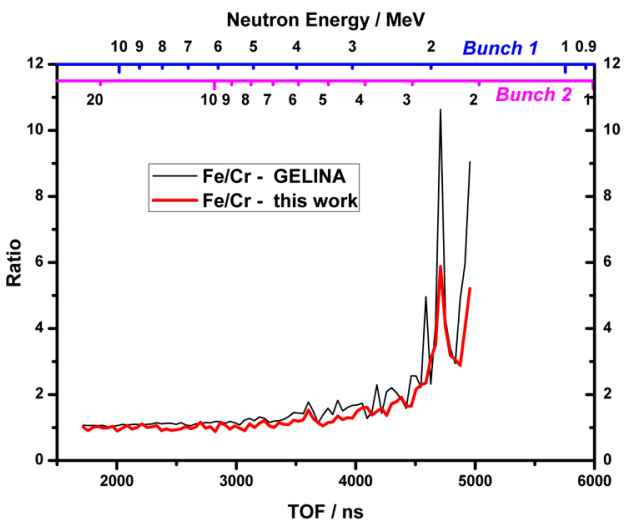

Figure 9. Comparision of the $\gamma$ emmisivity ratio of Fe-847keV and $\mathrm{Cr}-1434 \mathrm{keV}$ with GELINA.

\section{Conclusion}

In summary, we report new measurements of the inelastic neutron scatting on iron. By using the neutron facilities of Cockcraft-Walton Accelerator at CIAE, $2 \times 1.7 \mathrm{MV}$ accelerator at CIAE, and Back-n white neutron source at CSNS, the inelastic neutron scattering cross sections have been measured at 1.25, 2.45, 2.96 and $14.80 \mathrm{MeV}$. The results agree with these from other groups. To overcome the TOF blurring caused by the CSNS's double-bunch running mode, we are developing the unfolding method. Subsequently, we will measure more $(\mathrm{n}, \mathrm{xn} \gamma)$ cross sections for other significant nuclides.

\section{Acknowledgements}

This work was supported by the National Natural Science Foundation of China (No.11375275, 11790320, 1179032 1, 11790322), the continuous Basic Scientific Research Project (No. WDJC-2019-09).

\section{References}

[1] N.Fotiades, G.D.Johns, R.O.Nelson, et al., PHYSICAL REVIEW C 69, 024601 (2004)

[2] L.C.Mihailescu, L.Olah, C.Borcea, A.J.M.Plompen, Nuclear Instruments and Methods in Physics Research A 531, 375-391 (2004)

[3] D.Dashdorj, G.E.Mitchell, T.Kawano, et al., Nuclear Instruments and Methods in Physics Research B 261, 948-952 (2007)

[4] H.Chen, X.-L.Wang, Nat. Mater. 15, 689 (2016)

[5] Q.An et al., JINST 12, P07022 (2017)

[6] H.Yi et al, JINST 14, P02011 (2019) 